\title{
Contrast enhanced ultrasound in the assessment of urogenital pathology
}

\author{
Libero Barozzi ${ }^{1}$, Diana Capannelli ${ }^{2}$, Michele Imbriani ${ }^{1}$ \\ ${ }^{1}$ Department of Diagnostic Imaging, Radiology Unit, Maggiore Hospital, Bologna, Italy; \\ ${ }^{2}$ Cardio-Thoracic-Vascular Department, Radiology Unit, University of Bologna, Policlinico Sant'Orsola-Malpighi, Bologna, Italy.
}

\begin{abstract}
Summary Contrast enhanced ultrasound (CEUS) is an innovative technique that employs microbubble contrast agents to demonstrate parenchymal perfusion. Although initial clinical application was focused on the liver pathology, a wide variety of clinical conditions can be assessed now with CEUS. CEUS is a well-tolerated technique and is acquiring an increasing role in the assessment of renal pathology because contrast agents are not excreted by the kidney and do not affect the renal function.

CEUS demonstrated an accuracy similar to contrast enhanced multi-detector computed tomography (CEMDCT) in detecting focal lesions, with the advantage of the real-time assessment of microvascular perfusion by using time-intensity curves.

The aim of this paper is to review the main indications of CEUS in the assessment of renal and urogenital pathology. Imaging examples are presented and described. Advantages and limitations of CEUS with reference to conventional US and CE-MDCT are discussed.

KEY wORDS: Ultrasonography; Contrast Enhanced Ultrasonography; Urology.
\end{abstract}

Submitted 3 October 2014; Accepted 31 October 2014

\section{INTRODUCTION}

\section{CEUS}

Contrast enhancement ultrasound (CEUS) is a new technique that employs microbubble contrast agents and complementary harmonic pulse sequences to demonstrate parenchymal perfusion.

CEUS is widely employed in several fields of clinical practice The 2011 updated European Federation of Societies of Ultrasound in Medicine and Biology (EFSUMB) Guidelines and Recommendations on the Clinical Practice of CEUS have identified the current indications for the administration of US contrast agents for the study of different parts of the body, including the kidneys (1).

CEUS is useful in the detection and characterization of lesions, by differentiating solid neoplastic masses from pseudotumors or by graduating complex cystic lesions according with the Bosniak system $(2,3)$.
CEUS also allows evaluation of renal ischemia, infections and trauma.

CEUS allows a real-time multiplanar evaluation of microvasculature, which colour Doppler ultrasound cannot detect: it is useful to characterize the perfusional pattern of solid lesions (arterial and late phase). Other advantages of CEUS include its safety, simplicity, patient tolerance, lack of irradiation (conversely to CE-MDCT scans) (4-6). Microbubble contrast agents are not excreted by the kidney and do not affect renal function: they can be safely administrated to patients with renal insufficiency. Current contraindications are known hypersensitivity to any of the contrast agent components (even if ultrasound contrast agents have low rate of anaphylactic reactions) and recent acute cardiopulmonary diseases.

The main limit of this technique in the urinary tract assessment is that contrast agents are not concentrated in the collecting system and CEUS cannot give information about urinary excretory system. CEUS also have the same limitations of conventional ultrasound (US): poor sonic window due to bowel gas, ribs or patients with large body habitus (obesity) prevents good quality images. In these cases, CE-MDCT can give more information.

\section{Microbubble contrast agents}

Microbubble contrast agents consists of gas microbubbles (air or perfluorocarbon) stabilized by a biodegradable shell of protein, lipid or polymer. The small size of microbubbles (from 1 to 10 micrometres, as the size of a red blood cell) allows their passage unfiltered through the lungs but prevents entry into the interstitium allowing them to remain entirely intravascular ("pure blood pool" agents) (4-5, 7). Under US exposition, microbubbles oscillatory contract and expand themselves with the same resonance frequency of US waves, by amplifying the US signal. After circulating for several minutes, microbubbles dissolves: the gas is exhaled by the lungs whereas the biodegradable shell is metabolised by the liver.

\section{Technique}

Kidney has a single arterial blood supply, conversely to the liver. After endovascular bolus injection of the contrast agent, microbubbles diffuse to the blood pool. At first, it can be detected an arterial phase with corticomedullary differentiation lasting for 20-40 seconds. 
During the later phase $(45-120 \mathrm{sec})$, the enhancement is homogeneous and the differentiation between cortex and medulla is lost. The lesion contrast enhancement is evaluated in comparison with the surrounding parenchyma (4). Kidneys are highly vascularized and the contrast enhancement is faster than other abdominal organs: this allows the characterization of renal parenchyma but also the evaluation of liver (in the remaining 3 minutes after kidneys) and spleen (that retrains the contrast agent for as long as 7 minutes).

\section{KIDNEY}

\section{Renal infections}

The diagnosis of acute pyelonephritis is based on clinical evaluation and laboratory findings (1). Conventional baseline US demonstrates increased size of the kidney and cortical scarring, suggestive of previous episodes of infections. CEUS has an important role when the patient is still febrile after 72 hours despite of antibiotic treatment and a complicated pyelonephritis is suspected. As CE-MDCT, CEUS can show focal parenchymal areas of pyelonephritis that appear as wedge-shaped areas of reduced enhancement because of the parenchymal oedema (Figure 1).

Sometimes pyelonephritis can complicate with parenchymal abscessualization: a focal inhomogeneous nonenhancing area with intense peripheral uptake (Figure 2). Purulent material in pelvicalyceal system can be easily detected as echogenic material with no contrast uptake, since contrast agents are not concentrated in the collecting system. This finding is useful to differentiate pus from uro-endothelial tumours (5).

\section{Renal ischaemia}

Kidney has an abundant blood flow but can undergo a variety of vascular injuries.

CEUS demonstrates high accuracy in detecting kidney parenchymal ischaemia, comparable to CE-MDCT.

CEUS shows a higher sensibility in comparison to colour Doppler by detecting smaller blood vessels with slower blood flow.

Microbubbles reach the microvasculature and amplify the US signal, allowing a direct evaluation of parenchymal perfusion. Renal ischaemia appears as a triangular o wedge-shaped area with no contrast uptake, easily detectable in comparison to the surrounding normal parenchyma (Figure 3).

CEUS may also provide more precise information about tissue vitality: it can differentiate infarcts from areas of diminished perfusion. Even if both injuries appear at Colour Doppler as non-vascularized areas, the key finding is that only infarcts show complete lack of contrast enhancement after injection.

\section{Kidney transplant}

The renal transplant represent the ideal application of CEUS because the organ is superficial and well vascularized. Renal transplant can undergo a wide range of possible complications in the early post-operative period. The main important is the acute rejection.
Figure 1.

Contrast enhancement CT shows an inhomogeneous parenchymal enhancement due to the presence of parenchymal oedema (a). Baseline US (b) demonstrates a wedge-shaped ipoechogenic area with poor vascularization on Colour Doppler evaluation (c). CEUS confirms the lack of contrast enhancement in this area (d).

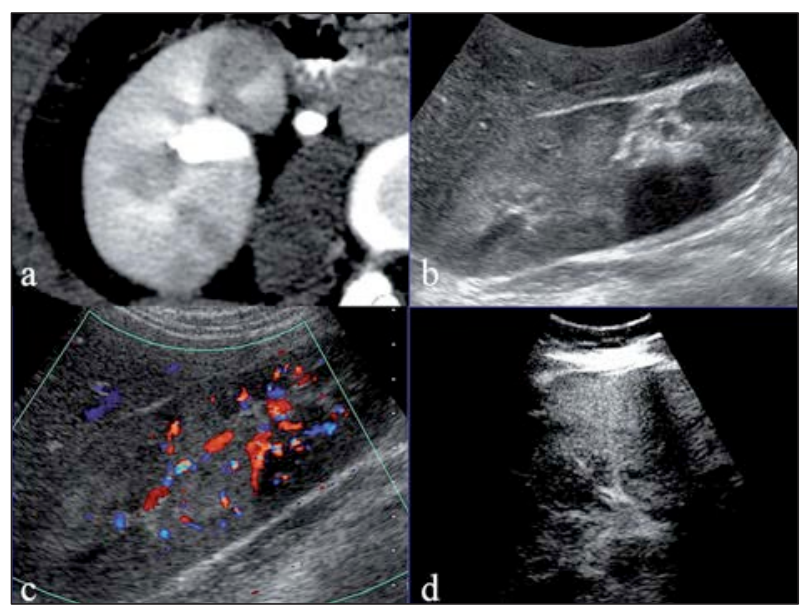

Figure 2.

Abscessualization. CEUS shows a poor-defined area of lack of contrast with an early and intense peripheral enhancement.

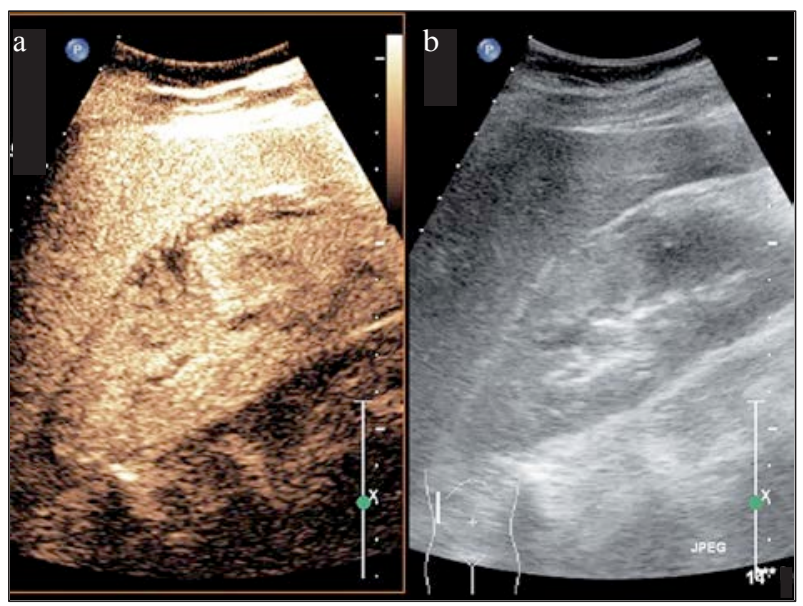

Figure 3.

Baseline US examination shows an ipoechogenic area involving the upper pole of the right kidney (a).

CEUS demonstrates a well-defined, triangular-shaped area of enhancement defect, suggestive of renal ischaemia (b).

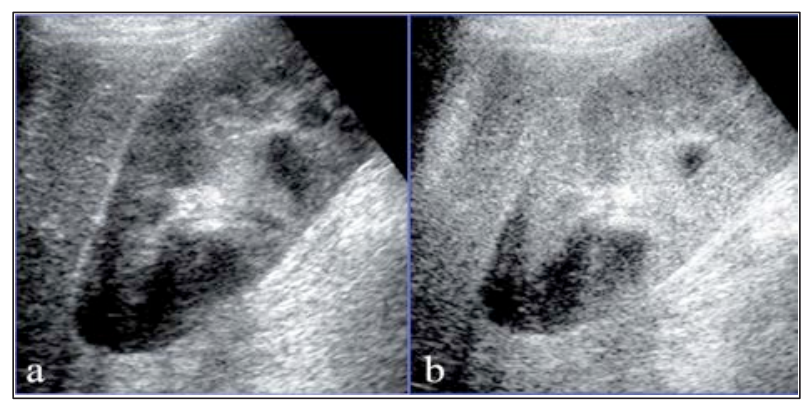

The first-line evaluation is typically performed with spectral Doppler measurements in order to assess abnormal values in resistance index (RI). 


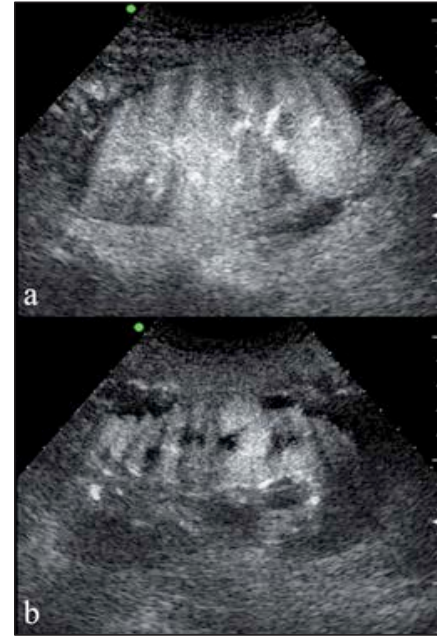

Figure 4

Normal kidney transplant: regular and homogeneous perfusion (a). Acute rejection in kidney transplant: CEUS demonstrates a dishomogeneous perfusion (b).

Spectral Doppler assessment only provide indirect information about the parenchymal perfusion, whereas microbubble contrast agents allow a direct visualization of microcirculation. CEUS findings are also earlier than abnormal RI (810). In acute rejection, the parenchymal perfusion is delayed. The time-intensity curves can demonstrate a diffuse delayed and slow contrast enhancement of the renal parenchyma. In a later phase, CEUS can also show perfusional defects (Figure 4). CEUS is also useful in monitoring the anti-rejection therapy, by assessing an improved parenchymal perfusion (11).

\section{Cystic lesions}

Renal cysts are a common finding, but any cyst that does not show the typical features of a benign cyst is by definition "complicated" and requires further assessment.

CEUS can be useful in differentiating benign cysts from cystic tumours. Even if the Bosniak classification system was developed on the basis of contrast-enhancement findings of cystic renal masses on CE-MDCT (2-3), CEUS can provide useful information for the management of these lesions: surgical treatment or observation. CEUS is acquiring an increasing role in the assessment of indeterminate cystic lesions (Bosniak IIF and III) by detecting the presence and the enhancement of solid components. Recent comparative studies (12) between CEUS and CT revealed that CEUS imaging was superior

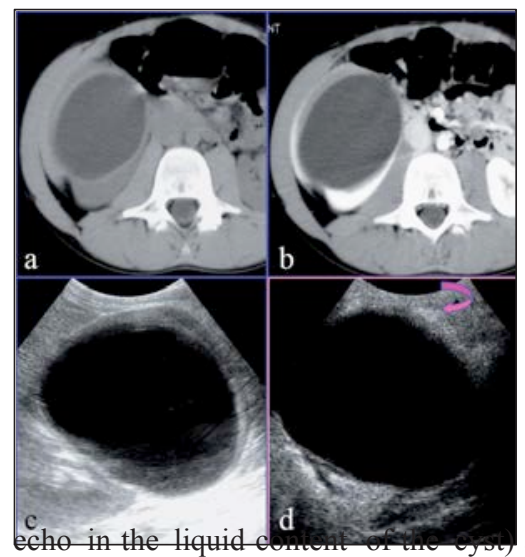

Figure 5. Contrast enhancement CT scan shows a complicated cystic lesion with grossly thicked walls (a), well marginated, without significant CE of the walls (b), suggestive of

Bosniak category III. Baseline US examination shows echoic content (solid/haemorrhagic and confirms the $\mathrm{CT}$ finding of thicked walls (c). CEUS demonstrates a wellenhancing mural nodule (arrow) within the lesion, suggestive of Bosniak IV category (d).
Figure 6.

Contrast enhancement CT scan shows a complicated cystic lesion with diffuse/smooth contrast enhancement (a). Colour Doppler examination demonstrate a heterogeneous mass with ipoechoic and fluid component without significant vascular apply (b). CEUS reveals an internal enhancing softtissue component, suggestive of Bosniak IV category (c).

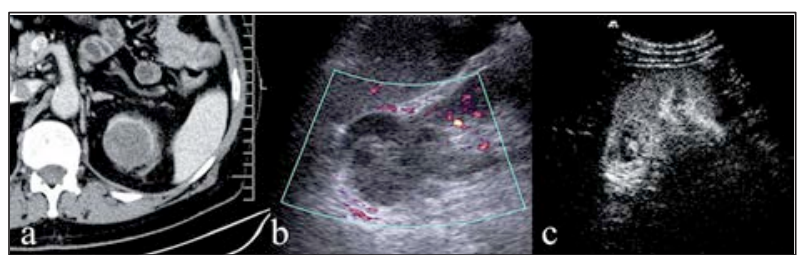

to CT in term of detecting additional septa, thickness of the wall or septa and solid components. Microbubble contrast agents circulate in the micro vessels of septa and walls and CEUS provides the evaluation of sophisticated internal structures of cystic renal masses with a higher resolution than $\mathrm{CT}$. In particular, the demonstration of solid components is the key factor in differential with the categories III and IV, that are considered malignant and must be surgically removed (Figures 5, 6).

\section{Solid masses}

The majority of renal tumours are renal cell carcinomas, whereas oncocytomas and angiomyolipomas represent a small part of renal solid lesions.

Renal malignancies have a rich blood supply and CEUS can show an increased and heterogeneous enhancement (Figure 7), fast filling and rapid wash-out (Figure 8). However, the kidney itself has abundant blood supply and the lesion may appear isoechoic to the surrounding renal cortex $(5,13)$.

CEUS is not currently used for differentiating between benign and malignant solid lesions. Even if several studies propose new methods for qualitative and quantitative assessment of contrast enhancement, solid malignancies does not show a specific perfusion pattern $(5,14)$.

CEUS may provide useful information in case of haemorrhage by detecting an underlined lesion into the haematoma that appear to conventional US evaluation as a large heterogeneous mass.

Another important role of CEUS is to differentiate pseudotumors (or renal column dysplasia) from solid malignan-

Figure 7.

Contrast enhancement CT scan shows a heterogeneous solid lesion, with intense peripheric enhancement (a). Colour Doppler examination shows an increased vascularization, both intralesional and peripherical (b). CEUS demonstrates an intense peripherical hyperenhancement, suggestive of renal clear cell carcinoma (c).

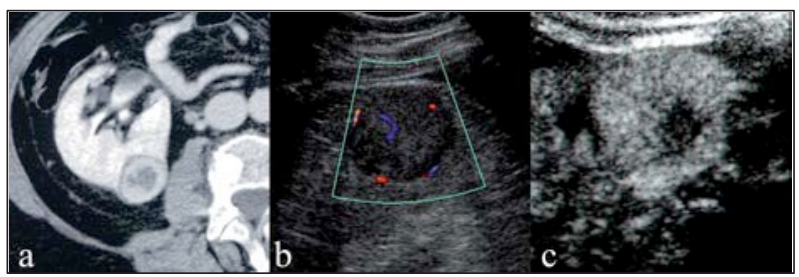


Figure 8.

Time-intensity curves display two different pattern of solid lesion contrast enhancement. In the first case (a), the red ROI was drawn in a suspicious area whereas the yellow ROI was drawn in an area representing the normal renal cortex. The red time-intensity curve shows a higher fast filling hyperenhancement with reference to the normal renal parenchyma, suggestive of renal clear cell carcinoma. Whereas in the second case (b), the red time-intensity curve show a later and lower enhancement with reference to the normal cortex, suggestive of ipovascularizated solid malignant lesion.

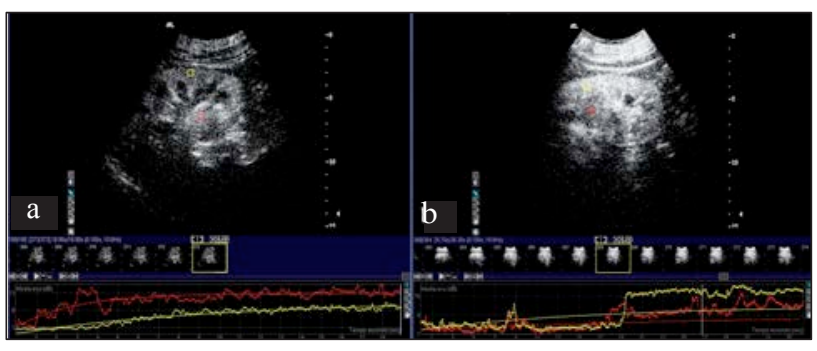

Figure 9.

Baseline US examination (a) shows an ipoechoic mass, suggestive of a solid occupying lesion. CEUS reveal a normal intravascular flow within this region, with homogeneous vascular enhancement without vessels distortion, suggestive of renal column dysplasia (b).

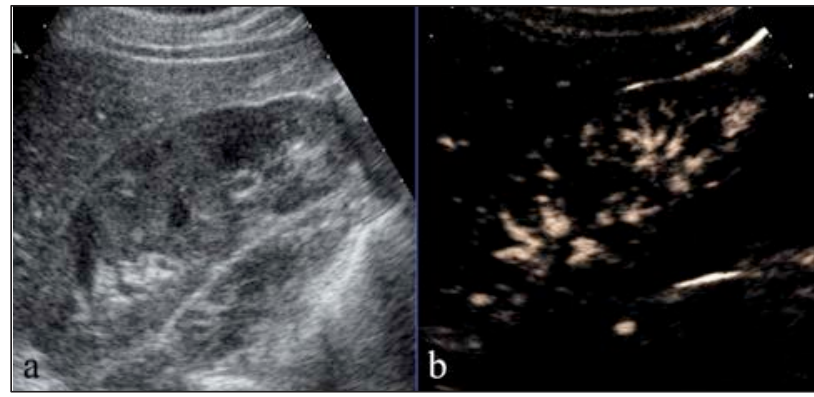

cies. Renal column hypertrophy is a congenital renal dysplasia that mimic a solid lesion of the cortex. CEUS demonstrates normal courses of renal vessels without an occupying lesion and a dynamic pattern of contrast enhancement identical to the surrounding parenchyma (Figure 9).

\section{Trauma}

CEUS with second-generation contrast agents shows a high sensitivity both in lesion detection and grading, but CEUS should be reserved for the assessment of stable, low-energy isolated trauma patients with unilateral pain. These patients have low risk for multi-organ and severe traumatic involvement, are haemodynamically stable and can be conservatively treated and evaluated during the follow-up (15-16).

Instead, the modality of choice for the first-line evaluation in emergency room of severe traumatic patients is the conventional FAST (Focus Assessment with Sonography in Trauma) US. FAST-US allows to exclude free abdominal, pleural and pericardial fluids, but it has low sensitivity in detection of parenchymal traumatic lesions, which may be isoechoic and can be missed (17).

CE-MDCT remains the reference examination in highenergy multitrauma because of high spatial resolution,
Figure 10.

Baseline US examination (a) is inadequate to detect renal laceration because it is isoechoic to the surrounding parenchyma. Colour Doppler (b) shows a relative homogeneous perfusion. After contrast injection, CEUS demonstrates a filling defect due to parenchymal laceration $(c, d)$.

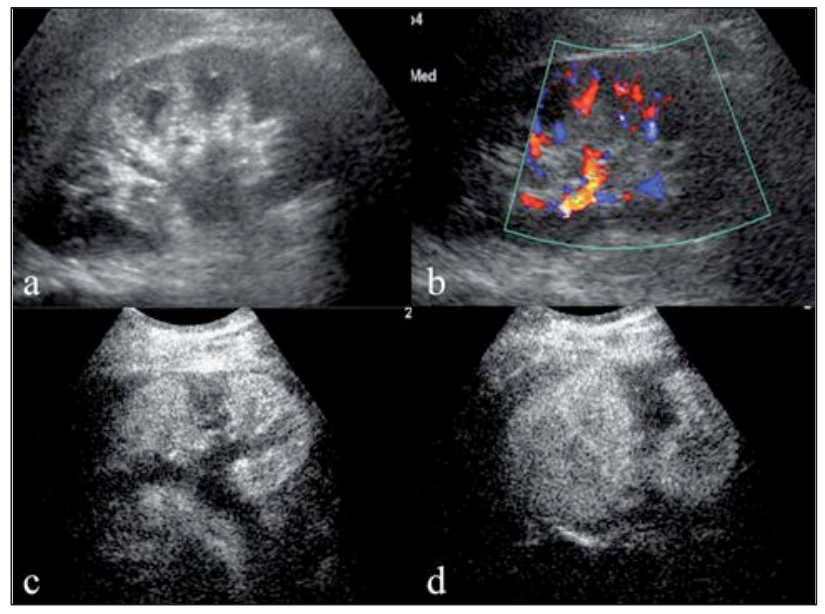

very fast execution and higher sensibility. CE-MDCT also allows to exclude active bleeding, multitraumatic involvement of deep organs (pancreatic trauma) and gut perforations. The main indication CEUS is in the secondline evaluation of patients with low-energy isolated abdominal trauma. CEUS demonstrates an accuracy similar to CE-MDCT in detecting and grading renal traumatic lesions. Parenchymal lacerations and haematomas appear as non-enhancing areas after contrast injection (Figure 10). The main limit of CEUS in kidney traumatic lesions is the impossibility to visualize pelvicalyceal and ureter injuries, since contrast agents are not concentrated in the collecting system. In these cases, CE-MDCT should be always performed in CEUS-positive patients to exclude active bleeding and urinomas.

\section{Urinary excretory system}

The main limit of CEUS in urinary excretory system is that contrast agents are not concentrated in the collecting system and only voluminous pelvicalyceal neoplastic masses can be detected.

CEUS is also acquiring an increasing role in the assessment of vesicoureteral reflux in children, because of the safety of the technique and the lack of irradiation (conversely to retrograde cystourethrography). After intrabladder administration of microbubble, CEUS is able to assess and quantify the grade of vesicoureteral reflux.

Urethra can also be involved in coital trauma. The intraurethral administration of microbubble constrast agent may improve the visualization of traumatic lesions or the detection of active urine extravasion.

\section{Prostate}

Trans-rectal CEUS has a high sensitivity in showing the cancer induced neovascularization and significantly improves ultrasound imaging for prostate cancer detection and localisation. In $85 \%$ of cases, prostate cancer is multifocal and it tends to grow along the capsule of the gland 


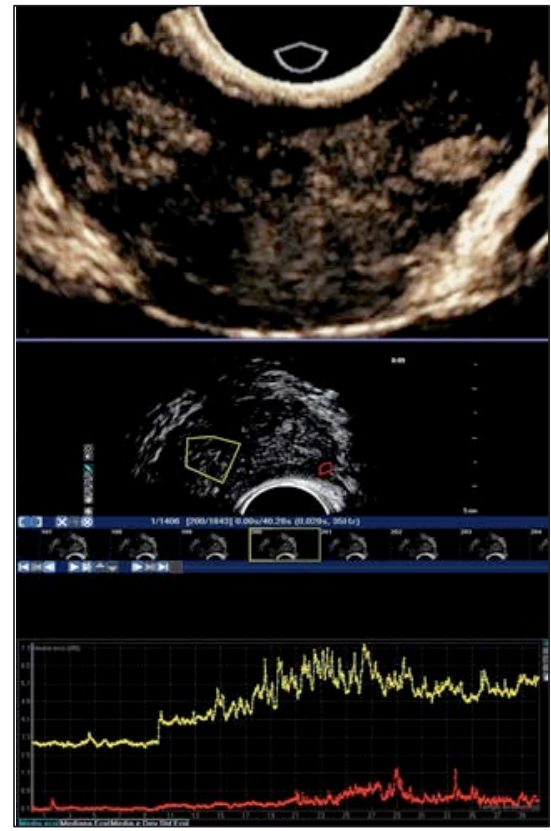

Figure 11.

Hipoechoic prostatic lesion on conventional trans-rectal US assessed with CEUS. The red ROI was drawn in a suspicious area whereas the yellow ROI was drawn in an area representing the normal prostatic parenchyma. The red time-intensity curve shows a poor contrast enhancement within the lesion, suggestive of hypovascularized prostatic tumour.

with an oblong shape. Prostate cancer differs from benign prostate tissue because of the loss of normal glandular architecture, increased cellular density and altered microvasculature. The loss of normal glandular architecture, characteristic of high-grade prostate cancer, results in fewer reflective interfaces and reduced echotexture on conventional ultrasound.

The classic gray scale ultrasound finding of cancer is a hypoechoic lesion (Figure 11). However, prostate cancer may appear echogenic or isoechoic and conventional US examination can miss them (18).

In these cases, CEUS can help in the detection of malignant lesions by demonstrating an area with early and increased enhancement, with reference to the surrounding parenchyma. The main differential diagnosis includes chronic prostatitis that can mimic the gray scale appearance of prostate cancer. Prostatitis may result in a heterogeneous appearance in the prostate peripheral zone and can present with hypoechoic lesions that are indistinguishable from cancer. CEUS evaluation with time-intensity curves may help in the differentiation.

CEUS also provides useful information to precisely target biopsies: a tailored approach to prostate biopsy based on contrast-enhanced ultrasound represents an innovative approach to detecting significant disease with fewer biopsy cores.

\section{Scrotum}

Acute scrotal pain is a challenging clinical problem that requires prompt diagnosis to determine appropriate treatment. It can be the result of a variety of causes, including torsion, epididymo-orchitis and tumours.

The first-line examination in patients with a painful scrotum is conventional US with Colour Doppler. According with the 2011 updated EFSUMB Guidelines (1), CEUS is acquiring an increasing role in the management of acute scrotal pain, because CEUS allow the correlation of macroscopic anomalies with possible perfusion alterations, providing a working diagnosis that would enable the urologist to pursue appropriate management: surgical treatment or observation.

In case of epididymitis or epididymo-orchitis, conventional US show a heterogeneous enlarged epididymis or testis with thickening. Both Colour Doppler and CEUS demonstrate increased vasculatization of the epididymis or testis. Sometimes inflammation can complicate with abscessualization. US tipically shows a sharp heterogeneous area without vascular signal inside the focal lesion on Colour Doppler. CEUS demonstrates focal absence of enhancement with hyperechoic peripheral rim.

CEUS aquired an important role in emergency practice also in the detection of testicular torsion. The testis appears on conventional US as a normal sized testicle with decreased flow on Colour Doppler. CEUS can shows the reduction or the complete absence of contrast enhancement of the testis, respectively in case of partial or complete torsion (Figure 12) (19).

CEUS is the imaging investigation of choice in case of scrotal trauma because it is able to depict parenchymal disorders on the basis of vascularity, helping in the differential diagnosis of scrotal lesions and traumatic changes. The key factor is to exclude testicular rupture and, in particular, the interruption of the tunica albuginea, allowing the urologist to decide when remove the injured testis or attempt salvage (15).

Testicular trauma appears as a hypoechoic interruption of the border of the testis with large surrounding haematoma. The integrity of the tunica albuginea may always be assessed. Intratesticular hyperechoic areas are suggestive of haemorragia.

In case of testicular rupture, CEUS demonstrates a lack of contrast enhancement in the hypoechoic area. CEUS can also detect active bleeding.

CEUS also help in detecting and characterizing testicular lesion of undefined nature at US.

Testicular neoplasms have a wide range of US presentation and the diagnosis is not possible on the basis of conventional US. Tumour can appear as heterogeneous hypoechoic area with poor vascularization on Colour Doppler. CEUS has a higher sensitivity in detecting ves-

Figure 12

Acute testicular torsion. Conventional US (b) shows a normal sized testicle. CEUS demonstrates the complete lack of contrast enhancement within the testis due to the absolute absence of testicular blood flow (a).

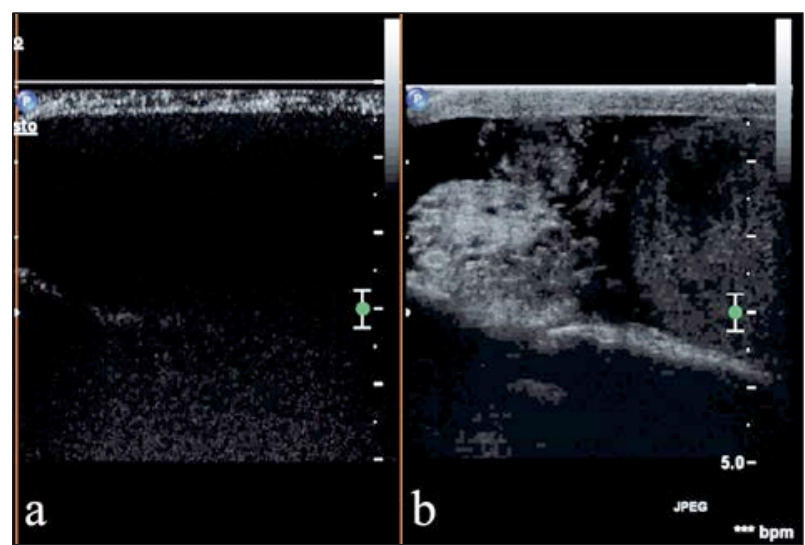


Figure 13.

Hypoechoic lesion on conventional US is assessed with CEUS. The red ROI was drawn in a suspicious area whereas the yellow ROI was drawn in an area representing the normal testicular parenchyma. The red time-intensity curve shows a higher fast filling hyperenhancement with reference to the normal testicular parenchyma and a rapid wash-out in the later phase, suggestive of testicular tumour.

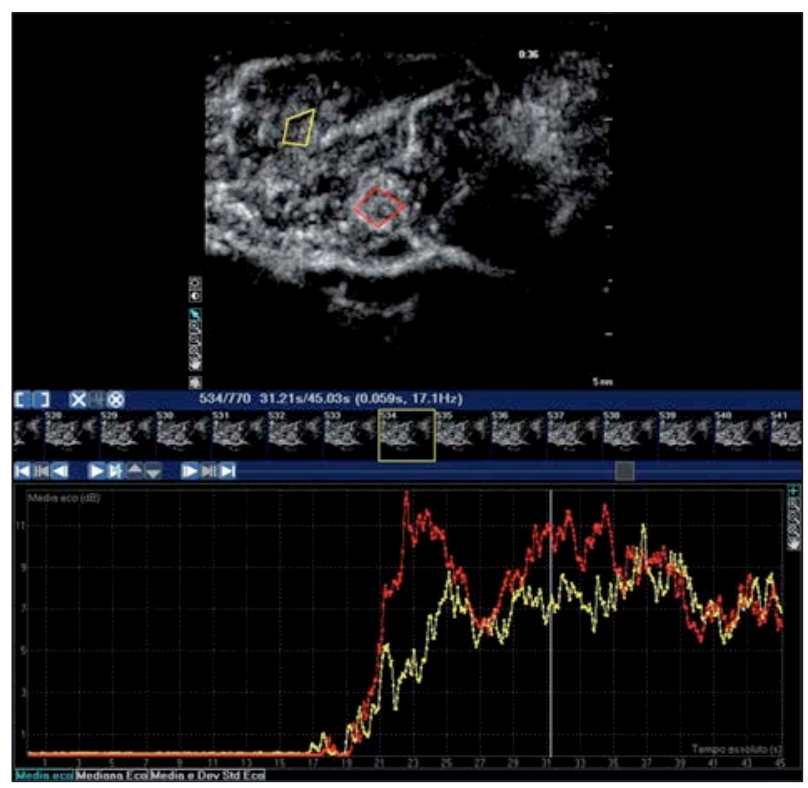

sels within the lesion and can show a slight or strong enhancement within the lesion in the early phase, that become hypoechoic compared to the surrounding parenchyma in the late phase. Time-intensity curves demonstrates an increased enhancement in early phase and rapid wash-out (Figure 13).

\section{Penis}

Sometimes penis can be involved in scrotal trauma, typically coital trauma. As for the testis, the key finding is the integrity of the tunica albuginea. The intracavernous administration of microbubble constrast agent may improve the visualization of traumatic lesions or the detection of active bleeding.

\section{REFERENCES}

1. Piscaglia F, Nolsøe C, Dietrich CF, et al. The EFSUMB Guidelines and Recommendations on the Clinical Practice of Contrast Enhanced Ultrasound (CEUS): update 2011 on non-hepatic applications. Ultraschall Med. 2012; 33:33

2. Bosniak MA. Diagnosis and management of patients with complicated cystic lesions of the kidney. AJR Am J Roentgenol. 1997; 169:819.

3. Bosniak MA.The use of the Bosniak classification system for renal cysts and cystic tumors. J Urol. 1997; 157:1852.

4. McArthur C, Baxter GM. Current and potential renal applications of contrast-enhanced ultrasound. Clin Radiol. 2012; 67:909.

5. Cokkinos DD, Antypa EG, Skilakaki M, et al. Contrast enhanced ultrasound of the kidneys: what is it capable of? Biomed Res Int. 2013; 59:5873

6. Jakobsen JA, Oyen R, Thomsen HS, Morcos SK. Members of
Contrast Media Safety Committee of European Society of Urogenital Radiology (ESUR). Safety of ultrasound contrast agents. Eur Radiol. 2005; 15:941.

7. Correas JM, Bridal L, Lesavre A, et al. Ultrasound contrast agents: properties, principles of action, tolerance, and artifacts. Eur Radiol. 2001; 11:1316.

8. Lebkowska U, Janica J, Lebkowski W, et al. Renal parenchyma perfusion spectrum and resistive index (RI) in ultrasound examinations with contrast medium in the early period after kidney transplantation.Transplant Proc. 2009; 41:3024.

9. Fischer T, Filimonow S, Dieckhöfer J, et al. Improved diagnosis of early kidney allograft dysfunction by ultrasound with echo enhancer-a new method for the diagnosis of renal perfusion. Nephrol Dial Transplant. 2006; 21:2921.

10. Granata A, Andrulli S, Fiorini F, et al. Diagnosis of acute pyelonephritis by contrast-enhanced ultrasonography in kidney transplant patients.Nephrol Dial Transplant. 2011; 26:715.

11. Fischer T, Mühler M, Kröncke TJ, et al. Early postoperative ultrasound of kidney transplants: evaluation of contrast medium dynamics using time-intensity curves. Rofo. 2004; 176:472.

12. Park BK, Kim B, Kim SH, et al. Assessment of cystic renal masses based on Bosniak classification: comparison of $\mathrm{CT}$ and contrastenhanced US. Eur J Radiol. 2007; 61:310.

13. Ignee A, Straub B, Schuessler G, Dietrich CF. Contrast enhanced ultrasound of renal masses. World J Radiol. 2010; 2:15.

14. Wang XH, Wang YJ, Lei CG. Evaluating the perfusion of occupying lesions of kidney and bladder with contrast-enhanced ultrasound. Clin Imaging. 2011; 35:447.

15. Valentino M, De Luca C, Barozzi L, et al. Contrast-enhanced US evaluation in patients with blunt abdominal trauma. J Ultrasound. $2010 ; 13: 22$.

16. Cokkinos DD, Antypa E, Kalogeropoulos I, et al. Contrastenhanced ultrasound performed under urgent conditions. Indications, review of the technique, clinical examples and limitations. Insights Imaging. 2013; 4:185

17. Smith ZA, Wood D. Emergency focused assessment with sonography in trauma (FAST) and haemodynamic stability. Emerg Med J. $2014 ; 31: 273$.

18. Mitterberger M, Pelzer A, Colleselli D, et al. Contrast-enhanced ultrasound for diagnosis of prostate cancer and kidney lesions. Eur J Radiol. 2007; 64:231.

19. Valentino M, Bertolotto M, Derchi L, et al. Role of contrast enhanced ultrasound in acute scrotal diseases. Eur Radiol. 2011; 21:1831.

\section{Correspondence}

Libero Barozzi, MD (Corresponding Author)

libero.barozzi@ausl.bologna.it

Michele Imbriani, MD

michele.imbriani@ausl.bologna.it

Department of Diagnostic Imaging, Radiology Unit, Maggiore Hospital Largo Nigrisoli 22 - 40100 Bologna, Italy

Diana Capannelli, MD

diana.capannelli@yahoo.it

Cardio-Thoracic-Vascular Department, Radiology Unit,

University of Bologna, Policlinico Sant'Orsola-Malpighi

Via Massarenti 9 - 40138 Bologna, Italy 